\title{
Electron pair current through the correlated quantum dot
}

\author{
T. Domański* and A. Donabidowicz \\ Institute of Physics, M. Curie Sktodowska University, 20-031 Lublin, Poland
}

(Dated: November 10, 2018)

\begin{abstract}
We study the charge current transmitted through the correlated quantum dot characterized by a finite magnitude of the Coulomb interaction $|U|$. At low temperatures the correlations can lead to a formation of the spin (for $U>0$ ) or charge (for $U<0$ ) Kondo states which qualitatively affect the transport properties. We explore the influence of charge Kondo effect on the electron pair tunneling introducing the auxiliary two-component model which describes fluctuations between the empty and doubly occupied states on QD.
\end{abstract}

\section{INTRODUCTION}

Electronic transport through the correlated quantum dots (QDs) has recently attracted a considerable interest both from a point of view of fundamental research as well as practical applications [1, 2]. Both these aspects are possible because of a large degree of flexibility for adjusting a coupling of QD to the external leads and a controlled positioning of the quantized QD levels by the gate voltage.

The tunability of the QD systems enabled controlled exploration of the Kondo effect [3], a phenomenon first observed in metals with diluted magnetic impurities [4]. At sufficiently low temperatures $T<T_{K}$ (where $T_{K}$ denotes the, so called, Kondo temperature) the spin of QD combines into a singlet state with the spins of itinerant electrons from the leads. In consequence the QD spectrum develops a narrow resonance at the chemical potential which at low temperatures enhances the zerobias conductance to the perfect unitary limit value $2 e^{2} / h$ [3]. On a microscopic level the underlying Kondo physics arises from an effective antiferromagnetic interaction between the QD and mobile electrons as envisaged by Schrieffer and Wolf within the perturbative canonical transformation [5].

Recently several authors [6, 7] have pointed out that molecular quantum dots affected by the bosonic degrees of freedom such as e.g. phonons could show up (besides the side-band structure) signatures of the charge Kondo effect. This phenomenon has been previously theoretically predicted for the heavy fermion compounds [8] and it might occur when the bipolaronic shift lowers the charging energy to a negative value $U<0$. The essential physics involved in the charge Kondo effect relies on a neutralization of the electron pair charge at the negative $U$ center (in the present context on QD) by electrons (or holes) from the adjacent leads. Due to a preferred double occupancy of QD there is activated a mechanism of the electron pair tunneling which manifests the qualitative features of charge Kondo effect in the differential conductance [7, 9] and in the thermopower [10].

\footnotetext{
${ }^{*}$ Corresponding author: doman@kft.umcs.lublin.pl
}

In what follows we propose a phenomenological twocomponent model which allows for a simple description of charge tunneling under the circumstances when the empty $|0\rangle$ and doubly occupied $|\uparrow \downarrow\rangle$ states are degenerate (which is a necesarry condition for realization of the charge Kondo effect [6, 7, 8] ). We discuss some preliminary results for the QD spectral function and the differential conductance obtained in the symmetric case using approximate treatment for the on-dot correlations.

\section{SPIN VERSUS CHARGE KONDO EFFECTS}

For a description of charge tunneling through the correlated quantum dot we use the Anderson model [4]

$$
\begin{aligned}
\hat{H}= & \sum_{\mathbf{k}, \beta, \sigma} \xi_{\mathbf{k} \beta} \hat{c}_{\mathbf{k} \beta \sigma}^{\dagger} \hat{c}_{\mathbf{k} \beta \sigma}+\hat{H}_{Q D} \\
& +\sum_{\mathbf{k}, \beta, \sigma}\left(V_{\mathbf{k} \beta} \hat{d}_{\sigma}^{\dagger} \hat{c}_{\mathbf{k} \beta \sigma}+V_{\mathbf{k} \beta}^{*} \hat{c}_{\mathbf{k} \beta \sigma}^{\dagger} \hat{d}_{\sigma}\right), \\
\hat{H}_{Q D}= & \sum_{\sigma} \varepsilon_{d} \hat{d}_{\sigma}^{\dagger} \hat{d}_{\sigma}+U \hat{d}_{\uparrow}^{\dagger} \hat{d}_{\uparrow} \hat{d}_{\downarrow}^{\dagger} \hat{d}_{\downarrow} .
\end{aligned}
$$

Operators $\hat{c}_{\mathbf{k} \beta \sigma}^{(\dagger)}$ correspond to annihilation (creation) of electrons in the left $\beta=L$ or right h.s. $\beta=R$ lead. The energies $\xi_{\mathbf{k} \beta \sigma}=\epsilon_{\mathbf{k} \sigma}-\mu_{\beta}$ are measured with respect to the chemical potentials which under nonequilibrium conditions are shifted by an applied bias $V$ through $\mu_{L}-\mu_{R}=e V$. The other terms containing $V_{\mathbf{k} \beta}^{(*)}$ describe hybridization of the QD to external leads. As usually $\hat{d}_{\sigma}^{(\dagger)}$ denote the annihilation (creation) of electron at the QD energy level $\varepsilon_{d}$ and $U$ is the on-dot Coulomb potential.

Let us first start by focusing on a widely studied case of the repulsive charging energy $U>0$. Conditions necesarry for a appearance of the Kondo effect can be explained using a perturbative treatment for the hybridization term $\hat{H}_{h y b}=\sum_{\mathbf{k}, \beta, \sigma}\left[V_{\mathbf{k} \beta} \hat{d}_{\sigma}^{\dagger} \hat{c}_{\mathbf{k} \beta \sigma}+\right.$ h.c. $]$. The unitary transformation $\hat{\tilde{H}}=e^{\hat{A}} \hat{H} e^{-\hat{A}}$ with the antihermitean generating operator $\hat{A}=\hat{\mathcal{A}}-\hat{\mathcal{A}}^{\dagger}$ where $\hat{\mathcal{A}}=\sum_{\mathbf{k}, \beta, \sigma} \frac{V_{\mathbf{k} \beta}}{\varepsilon_{d}-\xi_{\mathbf{k} \beta}}\left[\frac{U}{\varepsilon_{d}+U-\xi_{\mathbf{k} \beta}} \hat{d}_{-\sigma}^{\dagger} \hat{d}_{-\sigma}-1\right] \hat{c}_{\mathbf{k} \beta \sigma}^{\dagger} \hat{d}_{\sigma}$ eliminates $\hat{H}_{h y b}$ up to the quadratic terms [5]. Since for $U>0$ the double occupancy of QD is energetically expensive one can restrict to the subspace of singly occupied states 
when the transformed Hamiltonian reduces to the spin Kondo model [5]

$$
\hat{\tilde{H}}_{\text {spin }}^{\text {Kondo }}=\sum_{\mathbf{k}, \beta, \sigma} \xi_{\mathbf{k} \beta} \hat{c}_{\mathbf{k} \beta \sigma}^{+} \hat{c}_{\mathbf{k} \beta \sigma}-\sum_{\mathbf{k}, \mathbf{q}, \beta, \beta^{\prime}} J_{\mathbf{k}, \mathbf{q}}^{\beta, \beta^{\prime}} \hat{\vec{S}}_{d} \cdot \hat{\vec{S}}_{\mathbf{k} \beta, \mathbf{q} \beta^{\prime}}
$$

The QD spin operator $\hat{\vec{S}}_{d}$ can be conveniently expressed through $\hat{S}_{d}^{+}=\hat{d}_{\uparrow}^{\dagger} \hat{d}_{\downarrow}, \hat{S}_{d}^{-}=\hat{d}_{\downarrow}^{\dagger} \hat{d}_{\uparrow}$, and $\hat{S}_{d}^{z}=\frac{1}{2}\left(\hat{d}_{\uparrow}^{\dagger} \hat{d}_{\uparrow}-\hat{d}_{\downarrow}^{\dagger} \hat{d}_{\downarrow}\right)$ and similarly $\hat{S}_{\mathbf{k} \beta, \mathbf{q} \beta^{\prime}}^{+}=\hat{c}_{\mathbf{k} \beta \uparrow}^{\dagger} \hat{c}_{\mathbf{q} \beta \downarrow}$ etc. Near the Fermi surface the effective coupling $J_{\mathbf{k}, \mathbf{q}}^{\beta, \beta^{\prime}}$ simplifies to [5] $J_{\mathbf{k}_{F}, \mathbf{k}_{F}}^{\beta, \beta^{\prime}}=$ $\frac{U}{\varepsilon_{d}\left(\varepsilon_{d}+U\right)} V_{\mathbf{k}_{F} \beta} V_{\mathbf{k}_{F} \beta^{\prime}}^{*}$. In the regime of antiferromagnetic coupling $J_{\mathbf{k}_{F}, \mathbf{k}_{F}}^{\beta, \beta^{\prime}}<0$ and at sufficiently low temperatures $T<T_{K}$ the magnetic moment of $\mathrm{QD}$ is perfectly screened by spins of the itinerant electrons. For typical QDs the value of Kondo temperature $T_{K} \simeq \frac{\sqrt{U \Gamma}}{2} \exp \left\{\frac{\pi \varepsilon_{d}\left(\varepsilon_{d}+U\right)}{U \Gamma}\right\}$ (where $\Gamma \simeq 2 \pi \sum_{\beta}\left|V_{\mathbf{k}_{F} \beta}\right|^{2} \rho_{\beta}\left(\varepsilon_{F}\right)$ and $\rho_{\beta}\left(\varepsilon_{F}\right)$ is the density of states at the Fermi level) is of the order of hundreds mK. Appearance of such Kondo resonance pinned at the electrodes' chemical potentials enhances the zero bias conductance and this behavior has been indeed observed experimentally [3].

Formally for the negative $U$ model the canonical transformation can be done in the same way. Since the empty and doubly occupied QD states are then energetically more favorable therefore one focuses mainly on the terms describing pair tunneling $\sum_{\mathbf{k}, \mathbf{q}, \beta, \sigma, \sigma^{\prime}}\left(J_{\mathbf{k}, \mathbf{q}}^{\beta, \beta^{\prime}} \hat{d}_{\sigma}^{\dagger} \hat{d}_{-\sigma}^{\dagger} \hat{c}_{\mathbf{k} \beta-\sigma^{\prime}} \hat{\mathbf{c}}_{\mathbf{q} \beta^{\prime} \sigma^{\prime}}+\right.$ h.c. $)$ [7]. Taraphder and Coleman [8] have shown that in the symmetric case $\varepsilon_{d}+U / 2=0$ (here assuming also $V=0$ ) the attractive $U<0$ model becomes exactly isomorphic to its repulsive $U>0$ counterpart (1) under the following particle-hole (p-h) transformation $\hat{d}_{\downarrow}^{\dagger} \rightarrow-\hat{d}_{-\downarrow}, \hat{c}_{\mathbf{k} \beta \downarrow}^{\dagger} \rightarrow \hat{c}_{-\mathbf{k} \beta \downarrow}$. Outside the symmetric situation the p-h transformation still renders the structure of (1) with an additional Zeeman field $B^{z}=2 \varepsilon_{d}+U$ (see Ref. [7] for details). The effective Hamiltonian [5] operating on the relevant empty and doubly occupied QD states can be then expressed by [8]

$$
\begin{aligned}
\hat{\tilde{H}}_{\text {charge }}^{\text {Kondo }} & =\sum_{\mathbf{k}, \beta, \sigma} \xi_{\mathbf{k} \beta} \hat{c}_{\mathbf{k} \beta \sigma}^{+} \hat{c}_{\mathbf{k} \beta \sigma} \\
& +2 \sum_{\mathbf{k}, \mathbf{q}, \beta, \beta^{\prime}} J_{\mathbf{k}, \mathbf{q}}^{\beta, \beta^{\prime}} \hat{\overrightarrow{\mathcal{T}}}_{d} \cdot \hat{\overrightarrow{\mathcal{T}}}_{\mathbf{k} \beta, \mathbf{q} \beta^{\prime}}+\hat{\mathcal{T}}_{d}^{z} B^{z}
\end{aligned}
$$

where $\hat{\mathcal{T}}_{d}^{+}=\hat{d}_{\uparrow}^{\dagger} \hat{d}_{\downarrow}^{\dagger}, \hat{\mathcal{T}}_{d}^{-}=\hat{d}_{\downarrow} \hat{d}_{\uparrow}$, and $\hat{\mathcal{T}}_{d}^{z}=\frac{1}{2}\left(\hat{d}_{\uparrow}^{\dagger} \hat{d}_{\uparrow}+\hat{d}_{\downarrow}^{\dagger} \hat{d}_{\downarrow}-\right.$ 1). Using this pseudospin representation the spin Kondo effect can be directly translated into the charge Kondo effect of the model (4). The latter takes place in a vicinity of the degeneracy point $\varepsilon_{d}+U / 2 \sim 0$. So far some of its signatures in the pair tunneling through the QDs have been partly examined in Refs [6, 7].

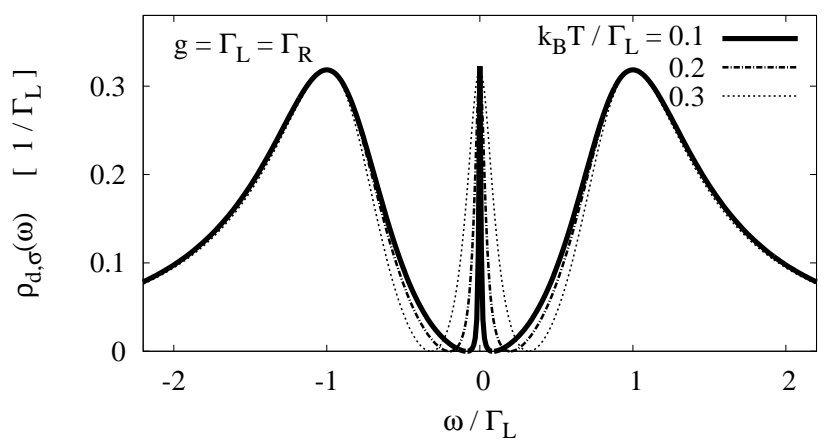

FIG. 1: Spectral function of the molecular quantum dot for the equilibrium situation $(V=0)$ in the symmetric case $E_{\text {pair }}=0, E_{d}=0$ for several temperatures. We used $g=\Gamma_{L}=\Gamma_{R}$ assuming the wide-band limit $\Gamma_{\beta}=0.001 D$, where $D$ is the bandwidth of conduction electrons.

\section{THE TWO-COMPONENT MODEL}

To consider the situation when the empty and doubly occupied states of $\mathrm{QD}$ are degenerate we introduce the following auxiliary model

$$
\begin{aligned}
\hat{H}_{Q D}= & \sum_{\sigma} E_{d} \hat{d}_{\sigma}^{\dagger} \hat{d}_{\sigma}+g\left(\hat{b}^{\dagger} \hat{d}_{\downarrow} \hat{d}_{\uparrow}+\hat{d}_{\uparrow}^{\dagger} \hat{d}_{\downarrow}^{\dagger} \hat{b}\right) \\
& +E_{\text {pair }} \hat{b}^{\dagger} \hat{b} .
\end{aligned}
$$

Whenever the electron pair happens to arrive on the dQD we let it be stored on the side-coupled buffer described by the operators $\hat{b}^{(\dagger)}$. They obey the hard-core boson relations [12] so that at most only one electron pair can be allocated on this side-coupled b-QD. Similar lattice version of this model has been proposed in the solid state physics to account for the bipolaron superfluidity in the crossover between the adiabatic and antiadiabatic regimes [12].

Loosely speaking the correspondence between (5) and the negative $U<0$ Hamiltonian (2) holds via substitutions $g=U, E_{\text {pair }}=2 \varepsilon_{d}+U$. This relation can be further supported analytically in the Lagrangian language after performing the Hubbard-Stratonovich transformation which eliminates $U \hat{d}_{\uparrow}^{\dagger} \hat{d}_{\uparrow} \hat{d}_{\downarrow}^{\dagger} \hat{d}_{\downarrow}$ through the additional bosonic fields, here denoted by $\hat{b}^{(\dagger)}$.

We will show that the two-component QD described by (5D) is able to capture the charge Kondo effect [8] known for the negative $U$ Anderson model [13] near the symmetric situation. The underlying mechanism is driven by suppressing the quantum fluctuations between the empty and doubly occupied states. Within the phenomenological model (5) such fluctuations are present even in the case of isolated molecular quantum dot i.e. for $V_{\mathbf{k} \beta}=0$. Out of 8 possible configurations being a product of the fermionic states $|0>,| \uparrow>,|\downarrow>,| \uparrow \downarrow>$ and the hard-core bosonic ones $\mid 0), \mid 1$ ) two of them $\uparrow \downarrow>\otimes \mid 0)$ and $|0>\otimes| 1)$ get mixed due to the Andreev-like interaction. A com- 
plete set of the eigenstates can be obtained using the transformation [14]

$$
\begin{aligned}
& |B>=\sin (\varphi)| 0>\otimes \mid 1)+\cos (\varphi)|\uparrow \downarrow>\otimes| 0), \\
& |A>=\cos (\varphi)| 0>\otimes \mid 1)-\sin (\varphi)|\uparrow \downarrow>\otimes| 0)
\end{aligned}
$$

with $\tan (2 \varphi)=\frac{2 g}{2 E_{d}-E_{\text {pair }}}$. For such limit $V_{\mathbf{k} \beta} \rightarrow 0$ the Green's function $\mathcal{G}_{d}(\omega)=\left\langle\left\langle\hat{d}_{\sigma} ; \hat{d}_{\sigma}^{\dagger}\right\rangle\right\rangle_{\omega}$ acquires the three pole structure [14]

$$
\mathcal{G}_{d}^{V_{\mathbf{k} \beta}=0}(\omega)=\frac{\mathcal{Z}}{\omega-E_{d}}+(1-\mathcal{Z})\left[\frac{u^{2}}{\omega-E_{+}}+\frac{v^{2}}{\omega-E_{-}}\right]
$$

where

$$
\begin{aligned}
v^{2}, u^{2} & =\frac{1}{2}\left(1 \mp \frac{1}{\gamma}\right) \\
E_{-}, E_{+} & =\frac{1}{2}\left[E_{\text {pair }} \mp\left(2 E_{d}-E_{\text {pair }}\right) \gamma\right]
\end{aligned}
$$

with $\gamma^{2}=1+4 g^{2} /\left(2 E_{d}-E_{\text {pair }}\right)^{2}$ and $\mathcal{Z}=$ $\left(1+e^{-E_{d} / k_{B} T}+e^{-\left(E_{d}+E_{\text {pair }}\right) / k_{B} T}+e^{-\left(2 E_{d}+E_{\text {pair }}\right) / k_{B} T}\right)$ $/\left[1+2 e^{-E_{d} / k_{B} T}+e^{-\left(E_{d}+E_{-}\right) / k_{B} T}+e^{-\left(E_{d}+E_{+}\right) / k_{B} T}+\right.$ $\left.2 e^{-\left(E_{d}+E_{\text {pair }}\right) / k_{B} T}+e^{-\left(2 E_{d}+E_{\text {pair }}\right) / k_{B} T}\right]$. The spectral weight $\mathcal{Z}$ of single particle level is gradually depleted for a decreasing temperature and its amount is transferred to the bonding $E_{-}$and antibonding $E_{+}$levels.

To get some insight into the many-body physics we employ a simple approximation based on the following substitution for the self-energy (see the discusion in Section 12.5 of the review book [2])

$$
\mathcal{G}_{d}(\omega)^{-1}=\mathcal{G}_{d}^{V_{\mathbf{k} \beta}=0}(\omega)^{-1}-\sum_{\mathbf{k}, \beta} \frac{\left|V_{\mathbf{k} \beta}\right|^{2}}{\omega-\varepsilon_{\mathbf{k} \beta}} .
$$

We shall focus on the wide-band limit when the hybridization couplings $\Gamma_{\beta}(\omega)=2 \pi \sum_{\mathbf{k}}\left|V_{\mathbf{k} \beta}\right|^{2} \delta\left(\omega-\varepsilon_{\mathbf{k} \beta}\right)$ can be treated as constant quantities $\Gamma_{\beta}$. Figure 1 shows the spectral function $\rho_{d, \sigma}(\omega)=-\frac{1}{\pi} \operatorname{Im} \mathcal{G}_{d}\left(\omega+i 0^{+}\right)$obtained for the symmetric case $E_{\text {pair }}=0, E_{d}=0$ when $u^{2}=0.5=v^{2}$ and $E_{+}=g, E_{-}=-g$. We notice the three-peak structure where the middle one is sensitive to temperature due to transfer of the spectral weight $\mathcal{Z}$. Let us emphasize that this behavior is typical for the Dicke effect known in quantum optics where the narrow/broad energy features correspond to the states weakly/strongly coupled to the electromagnetic field and they contribute the subradiant/superradiant emission lines [15]. Recently a similar concept of the Kondo-Dicke effect has been proposed in mesoscopic physics for a set of three vertically coupled quantum dots [16]. Our proposal (5) formally belongs to the same class and some details concerning its relation to the Dicke effect have been already in pointed out in the review paper [15].

For the present context it is important to emphasize that the spectral function $\rho_{d, \sigma}(\omega=0)$ is temperatureindependent. In order to clarify this property let us consider the bare Green's function (8) which at low energies

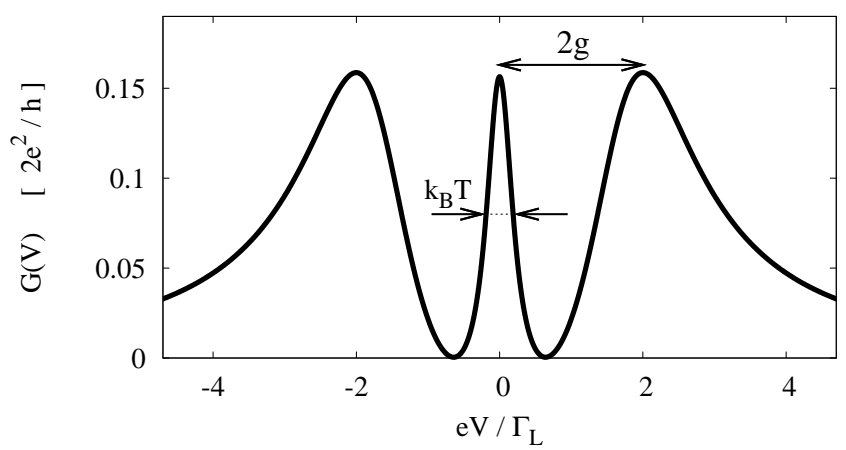

FIG. 2: The differential conductance $G(V)$ as a function of bias voltage $V$ in the symmetric case for $k_{B} T=0.2 \Gamma_{L}, \Gamma_{R}=$ $\Gamma_{L}$ and $g=\Gamma_{L}$. Width of the central (superradiance) peak is proportional to temperature whereas the zero bias value $G(0)$ remains constant (temperature-independent) 7].

$\omega \rightarrow 0$ simplifies to $G_{d}^{V_{\mathbf{k} \beta}=0}(\omega) \simeq \mathcal{Z} / \omega$ (we refer here to the symmetric case $E_{d}=0$ ). Using the Ansatz (11) we obtain $\mathcal{G}_{d}(\omega) \simeq \frac{\mathcal{Z}}{\omega+i \mathcal{Z}\left(\Gamma_{L}+\Gamma_{R}\right) / 2}$ which yields the following fixed value of the spectral function $\rho_{d, \sigma}(\omega) \simeq \frac{1}{\pi} \frac{2}{\Gamma_{L}+\Gamma_{R}}$. Furthermore we notice that effective broadening of a central peak depends on the spectral weight $\mathcal{Z}$, hence comes its temperature dependence which is illustrated in figure 1. Obviously, in a weak interaction regime $|g| \ll \Gamma_{\beta}$ the three peaks overlap with one another therefore the fine structure related to the charge Kondo state fades away.

\section{TRANSPORT PROPERTIES}

External bias $V$ applied between the electrodes induces the charge and energy currents through the interface. We expect that behavior presented in figure 11 is going to have an impact on the low-temperature transport properties. To determine the charge current $I_{\beta}(V)=-e \frac{d}{d t}\left\langle\sum_{\mathbf{k} \sigma} \hat{c}_{\mathbf{k} \beta \sigma}^{+} \hat{c}_{\mathbf{k} \beta \sigma}\right\rangle$ one has to use either the nonequilibrium Keldysh or Kadanoff-Baym formalism [1]. Standard calculations [1] yield the steady electron current $I(V)=I_{L}(V)=-I_{R}(V)$ given by the Landauertype formula

$$
I(V)=\frac{2 e}{h} \int_{-\infty}^{\infty} d \omega\left[f\left(\omega-\mu_{L}\right)-f\left(\omega-\mu_{R}\right)\right] T(\omega),
$$

where $\mu_{L}-\mu_{R}=e V$ and $f(\omega)=\left[1+\exp (\omega) / k_{B} T\right]^{-1}$. Correlation effects show up within such approach through the transmission coefficient $T(\omega)=\frac{\Gamma_{L} \Gamma_{R}}{\Gamma_{L}+\Gamma_{R}} \sum_{\sigma} \rho_{d, \sigma}(\omega)$. This treatment is valid for the wide-band limit $\Gamma_{\beta} \ll D$ and it can be derived, for instance from the equation of motion method [17].

We have explored numerically the differential conductance $G(V)=\frac{d}{d V} I(V)$ for a number of temperatures. Figure 2 shows a representative result obtained for $k_{B} T=0.2 \Gamma_{L}$ where a middle peak corresponds to 
the characteristic charge Kondo feature. Its width is proportional to temperature, whereas the zero bias conductance $G(0)=\frac{2 e^{2}}{h} \int_{-\infty}^{\infty} d \omega\left[-\frac{d f(\omega)}{d \omega}\right] T(\omega)$ at low temperatures approaches the asymptotic value $\lim _{T \rightarrow 0} G(0)=$ $\frac{2 e^{2}}{h} \frac{\Gamma_{L} \Gamma_{R}}{\Gamma_{L}+\Gamma_{R}} \sum_{\sigma} \rho_{d, \sigma}(0)$. In the previous study [7] of the charge Kondo physics such feature has been predicted for the zero-bias conductance as a function of the gate voltage. From our study of the two-component model (5) we find the same behavior but this issue will be discussed separately.

\section{SUMMARY}

We have studied the charge transport through a correlated quantum dot with an effective negative value of the Coulomb interaction $U<0$. To account for the quantum fluctuations between the empty and doubly occupied states we have introduced the phenomenological model where QD dot is coupled to an additional electron pair buffer via the Andreev-type interaction. Focusing on the symmetric case, when the Kondo effect arises in the pseudospin charge channel [8], we have examined its influence on the spectral function and the differential conductance. For both these quantities we have found the narrow peak whose height is constant whereas its width is proportional to temperature. This resembles the properties of the Dicke effect [15] which has been recently considered for a configuration composed of the three vertically coupled quantum dots [16]. It might be of some interest to proceed with an analysis of here proposed model outside the symmetric case and adopt some more sophisticated selfconsistent treatment for the Andreev-like interaction on the molecular quantum dot.

This work is partly supported by the Polish Ministry of Science and Education under the grants NN202187833 and NN202373333.
[1] I.L. Aleiner, P.W. Brouwer, and L.I. Glazman, Phys. Rep. 358, 309 (2002).

[2] H. Haug, A.P. Yauho, Quantum Kinetics in Transport and Optics of Semiconductors, Springer-Verlag, Berlin (1996).

[3] D. Goldhaber-Gordon, H. Shtrikman, D. Mahalu, D. Abusch-Magder, U. Mairav, and M.A. Kastner, Nature 391, 156 (1998); W.G. van der Wiel, S. De Franceschi, T. Fujisawa, J.M. Elzerman, S. Tarucha, L.P. Kouwenhoven, Science 289, 2105 (2000).

[4] A.C. Hewson, The Kondo problem to Heavy Fermions Cambridge University Press, Cambridge, (1993).

[5] J.R. Schrieffer, P.A. Wolf, Phys. Rev. 149, 491 (1966).

[6] J. Mravlje, A. Ramsak, and T. Rejec, Phys. Rev. B 72, 121403 (2005); L. Arachea and M.J. Rozenberg, Phys. Rev. B 72, 041301 (2005); P.S. Cornaglia, H. Ness, and D.R. Grempel, Phys. Rev. Lett. 93147201 (2003); A.S. Alexandrov and A.M. Bratkovsky, Phys. Rev. B 67, 235312 (2003); A.S. Alexandrov, A.M. Bratkovsky, and R.S. Williams, Phys. Rev. B 67, 075301 (2003).

[7] J. Koch, E. Sela, Y. Oreg, and F. von Oppen, Phys. Rev. B 75, 195402 (2007); J. Koch, M.E. Raikh, and F. von
Oppen, Phys. Rev. Lett. 96, 056803 (2006).

[8] A. Taraphder and P. Coleman, Phys. Rev. Lett. 66, 2814 (1991).

[9] M.J. Hwang, M.S. Choi, and R. Lopez, Phys. Rev. B 76, 165312 (2007).

[10] M. Gierczak and K.I. Wysokiński, J. Phys.: Conf. Ser. 104, 012005 (2008).

[11] Y. Meir, N.S. Wingreen, P.A. Lee, Phys. Rev. Lett. 70, 2601 (1993).

[12] J. Ranninger and S. Robaszkiewicz, Physica B 135, 468 (1985).

[13] P.W. Anderson, Phys. Rev. Lett. 34, 953 (1975).

[14] T. Domański, J. Ranninger, and J.M. Robin, Solid State Commun. 105, 473 (1998); T. Domański, Eur. Phys. J. B 33, 41 (2003).

[15] T. Brandes, Phys. Rep. 408, 315 (2004).

[16] P. Trocha and J. Barnaś, Phys. Rev. B 78, 075424 (2008); P. Trocha and J. Barnaś, J. Phys.: Condens. Matter 20, 125220 (2008).

[17] T.-K. Ng, Phys. Rev. Lett. 76, 487 (1996). 